\title{
Efecto del tipo de promotor de crecimiento sobre el rendimiento productivo en pollos de engorde, bajo condiciones de trópico
}

\author{
Effect of the growth promoter type on the productive yield in broilers, under tropical \\ conditions
}

\author{
M.A. Urbano ${ }^{1}$, C. R. Velásquez ${ }^{1,2}$
}

\begin{abstract}
Resumen
Objetivos:Determinar el efecto del tipo de promotor de crecimiento sobre el rendimiento productivo en pollos de engorde, bajo condiciones de trópico. Metodología: La investigación se realizó en una granja comercial de pollos de engorde ubicada en la selva central de Ucayali, Pucallpa, Provincia de Coronel Portillo. Se evaluaron 150 pollitos de un día de nacido machos de la línea Cobb 500, que fueron distribuidos al azar en tres grupos de 50 pollos, cinco réplicas y 10 pollos por réplica. Los tratamientos fueron: $\mathrm{T}_{0}$ : Antibióticos, $\mathrm{T}_{1}$ : Probióticos y $\mathrm{T}_{2}$ : Orégano seco molido $1 \%$. Las variables evaluadas fueron: peso vivo (PV), consumo de alimento (CA) y conversión alimenticia (ECA). Se utilizó el diseño completamente al azar, los datos se analizaron con el ANVA y para la comparación de medias, la prueba de Tukey. Resultados: Al comparar los antibióticos con los probióticos no se encontraron diferencias significativas $(P>0,05)$ en el PV $(2,62 ; 2,57 \mathrm{~kg}), \mathrm{CA}(4,75 ; 4,71 \mathrm{~kg})$ y ECA $(1,81 ; 1,83)$. El tratamiento con orégano al $1 \%$ tuvo un menor PV $(2,44 \mathrm{~kg})(P<0,05)$ y similar $(P>0,05)$ CA $(4,49 \mathrm{~kg})$ y ECA $(1,83)$ en comparación a los demás tratamientos. Conclusiones: Las dietas alimenticias con antibióticos y probióticos tuvieron un mejor rendimiento en peso vivo en comparación al $1 \%$ de orégano.
\end{abstract}

Palabras clave: Antibiótico, probiótico, orégano, pollos de engorde, promotores de crecimiento

\begin{abstract}
Objectives: To determine the effect of the type of growth promoter on the productive yield in broilers, under tropical conditions. Metodología: The research was carried out in a commercial broiler farm located in the central jungle of Ucayali, Pucallpa, Coronel Portillo Province. One hundred fifty one-day-old male chicks of the Cobb 500 line were evaluated, which were randomly distributed in three groups of 50 chickens, 05 repetitions and 10 chickens per repetition. The treatments were: $\mathrm{T}_{0}$ : Antibiotics, $\mathrm{T}_{1}$ : Probiotics and $\mathrm{T}_{2}: 1 \%$ ground dried oregano. The variables evaluated were: Live weight (PV), Food consumption (CA) and Food conversion (ECA). The data were analyzed with the ANOVA and the Tukey test to determine differences between the averages of the treatments. Results: When comparing $\mathrm{T}_{0}$ (antibiotics) with $\mathrm{T}_{1}$ (probiotics), no significant differences $(P>0.05)$ were found in the PV $(2.62,2.57 \mathrm{~kg}), \mathrm{CA}(4.75,4.71 \mathrm{~kg})$ and ECA $(1.81,1.83)$. The $\mathrm{T}_{2}$ (oregano $\left.1 \%\right)$ had a lower PV $(2.44 \mathrm{~kg})(P<0.05)$ and similar $(P>0.05) \mathrm{CA}(4.49 \mathrm{~kg})$ and ECA $(1.83)$ compared to $\mathrm{T}_{0}$ and $\mathrm{T}_{1}$. Conclusions: Diets with antibiotics and probiotics had a better productive yield compared to oregano $1 \%$.
\end{abstract}

Keywords: Antibiotic, probiotic, oregano, broilers, growth promoters

\footnotetext{
${ }^{1}$ Departamento de Zootecnia, Universidad Nacional José Faustino Sánchez Carrión, Perú.

${ }^{2}$ Autor para correspondencia: cvelasquez@unjfsc.edu.pe
} 


\section{Introducción}

La producción avícola en Perú registra un crecimiento sostenido en los últimos años; en el 2018, el valor bruto de la producción avícola ascendió a 9140 millones de soles, que representa un crecimiento del 7,7 \% con relación al 2017, este incremento estuvo influenciado principalmente en el aumento en la producción de pollo $(8 \%)$ y gallina de postura $(7,7 \%)$ (Ministerio de Agricultura y Riego, 2019).

El uso de antibióticos promotores del crecimiento(APC)en laalimentación contribuyen a optimizar la eficiencia productiva en las aves, por sus beneficios sobre la salud intestinal. Sin embargo, la comunidad europea desde hace 12 años ha prohibido el uso de antibióticos en la alimentación del ganado en todos los países socios, por contribuir a la resistencia bacteriana, lo cual afecta el tratamiento con antibióticos de enfermedades infecciosas en los animales y el hombre (Diario Oficial de la Unión Europea, 2003).

Esta prohibición se viene extendiendo gradualmente a todos los países. Ante esta situación, las investigaciones por encontrar aditivos que sustituyan de manera eficaz a los antibióticos se han incrementado (Gaggia et al., 2010). Los probióticos se han consolidado como una estrategia terapéutica de origen nutricional al uso de los APC. Los probióticos no generan efectos colaterales y mejoran la digestibilidad, ganancia en peso y conversión alimentaria (Quigley, 2010).

El uso de plantas con propiedades medicinales, como el orégano, puede ser una alternativa de solución para reemplazar a los APC. Actualmente, se reconocen múltiples efectos funcionales de las plantas medicinales, en el caso del orégano se conoce que tienen propiedades antimicrobianas in vitro, antioxidantes, antimicóticos, antiparasitarios y estimulantes de la secreción de enzimas digestivas. El orégano surge entonces como una alternativa integral para su uso como pro nutrientes en la alimentación animal (Hernández et al., 2004).

La presente investigación evaluó el efecto del tipo de promotor de crecimiento sobre el rendimiento productivo en pollos de carne, bajo condiciones de trópico.

\section{Metodología}

La investigación se realizó en una granja comercial de pollos de engorde ubicada en la selva central de Ucayali, Pucallpa, Provincia de Coronel Portillo km 6 de la carretera marginal Jorge Basadre, para tal fin, se adecuó un galpón de pollos con 15 divisiones internas de $1 \mathrm{~m} 2$ con capacidad para 10 pollos.

Se utilizaron 150 pollitos BB machos de la línea Cobb 500, que fueron distribuidos al azar en tres grupos de 50 individuos. A cada grupo se le asignó un tratamiento con cinco replicaciones de 10 pollitos cada uno.

Todos los pollos de los tres tratamientos recibieron la misma dieta alimenticia en todas las etapas de crianza (Pre Inicio, inicio, crecimiento y acabado) y fueron vacunados, en el agua de bebida, contra el Newcastle - Bronquitis infecciosa (Día 6) y Gumboro (Día 12).

Los tratamientos evaluados fueron: $\mathrm{T}_{0}$ : Antibióticos (Neomicina, Lincomicina $\mathrm{y}$ Bacitracina); $\mathrm{T}_{1}$ : Probiótico (Lactobacillus acidophilus) y $\mathrm{T}_{2}$ : Orégano seco molido $1 \%$.

Las variables en estudio fueron: Peso vivo, consumo de alimento, conversión alimenticia y se evaluaron hasta la sexta semana de vida.

Los datos obtenidos se recolectaron en una hoja de cálculo Excel y fueron analizados con el programa Minitab v.18. Los datos que cumplieron con los criterios de normalidad e igualdad de varianzas fueron analizados con el ANVA y la prueba de Tukey se utilizó para determinar diferencias entre los promedios de los tratamientos.

\section{Resultados y discusión}

\section{Peso vivo}

Las aves tratadas con antibióticos $\left(\mathrm{T}_{0}\right)$ alcanzaron un PV (2 623,2 g) similar $(P>$ $0,05)$ al obtenido con probióticos $\left(\mathrm{T}_{1}\right)$ (2 578 g) y mayor $(P<0,05)$ al obtenido con orégano 
( $\mathrm{T}_{2}$ ) (2 $\left.445 \mathrm{~g}\right)$. Los pollos del tratamiento con antibióticos $\left(\mathrm{T}_{0}\right)$ tuvieron un PV mayor en $1,7 \%$ al conseguido con probióticos $\left(\mathrm{T}_{1}\right)$ y $7,2 \%$ mayor que el orégano $1 \%\left(\mathrm{~T}_{2}\right)$ (Tabla 1$)$.

\section{Consumo de alimento}

No se observaron diferencias significativas en la CA $(P>0,05)$ al comparar los tres tratamientos. Sin embargo, los pollos del tratamiento con antibióticos $\left(\mathrm{T}_{0}\right)$ consumieron $2 \%$ más de alimento en comparación a los probióticos $\left(\mathrm{T}_{1}\right) \mathrm{y}$ $6 \%$ más que el orégano $1 \%\left(\mathrm{~T}_{2}\right)$ (Tabla 1$)$.

\section{Conversión alimenticia}

No se observaron diferencias significativas $(P>0,05)$ en la ECA de los pollos de carne. La ECA del grupo de aves alimentadas con antibióticos $\left(\mathrm{T}_{0}\right)$ fue $1 \%$ mayor al grupo de aves alimentadas con Probióticos $\left(\mathrm{T}_{1}\right)$ y con orégano 1\% (Tabla 1).

En el estudio, las aves alimentadas con antibióticos (T0), registraron similar PV, CA y ECA al obtenido en T1 (Probióticos). Estos resultados concuerdan con los obtenidos por Osorio et al. (2010) quienes, al comparar el uso de un antibiótico con un probiótico en aves, no encontraron diferencias $(P>0,05)$ entre estas variables. Los resultados de este estudio coinciden con otros que usaron cepas de Lactobacillus (Watkins \& Kratzer, 1984),
Enterococcus faecium (Chávez et al., 2016), Bifidobacterium bifidum (Loddi et al., 2000) y mezcla de bacterias ácido lácticas en el agua de bebida (Rincón et al., 2000).

Los tratamientos con antibióticos $\left(\mathrm{T}_{0}\right) \mathrm{y}$ probióticos $\left(\mathrm{T}_{1}\right)$ registraron un mayor $\mathrm{PV}$, con similares CA y de ECA en comparación al $\mathrm{T}_{0}$ (orégano 1\%). Estos resultados son similares a los obtenidos por Ascención (2011) quien obtuvo un mayor peso vivo al comparar el uso de diferentes antibióticos con el orégano en la dieta alimenticia de pollos de engorde. Así mismo, los resultados no concuerdan con los obtenidos por Lara y Lara et al. (2010) quienes no encontraron diferencias $(P>0,05)$ al comparar el uso del antibiótico flavomicina con el orégano.

Los mejores resultados obtenidos con los antibióticos sobre las variables productivas evaluadas, se deberían al mejor control que ejerce sobre la flora intestinal patógena permitiendo el establecimiento de flora benéfica (Jernigan et al., 1985; Salas, 2005) que favorece un mejor aprovechamiento de los nutrientes. En el caso de los probióticos, el efecto benéfico se debería por el mantenimiento de la microbiota intestinal normal por exclusión competitiva y antagonismo (Quigley, 2010). Con relación al orégano, si bien tiene propiedades antimicrobianas que mejoran la salud intestinal del ave, sin embargo, los conocimientos de las propiedades benéficas de esta planta no son bien conocidos; por esta razón, los resultados obtenidos por diversos

\section{Tabla 1}

Parámetros productivos en pollos de carne, a los 42 días de edad, como respuesta al uso de probióticos, antibióticos y orégano 1\%

\begin{tabular}{|c|c|c|c|}
\hline Tratamientos & Peso vivo & Consumo de Alimento & Conversión Alimenticia \\
\hline & (g) & (g) & $(g / g)$ \\
\hline $\mathrm{T}_{0}:$ Antibióticos & $2623,2 \pm 26,0^{\mathrm{a}}$ & $4759,0 \pm 119,0^{\mathrm{a}}$ & $1,81 \pm 0,04^{\mathrm{a}}$ \\
\hline $\mathrm{T}_{1}:$ Probióticos & $2578,0 \pm 24,6^{\mathrm{a}}$ & $4716,0 \pm 95,0^{\mathrm{a}}$ & $1,83 \pm 0,04^{\mathrm{a}}$ \\
\hline $\mathrm{T}_{2}:$ Orégano $1 \%$ & $2445,4 \pm 43,7^{b}$ & $4493,8 \pm 62,2^{\mathrm{a}}$ & $1,83 \pm 0,07^{\mathrm{a}}$ \\
\hline
\end{tabular}

${ }^{\mathrm{a}, \mathrm{b}}$ Letras diferentes indican signficancia estadistica $(P<0,05)$. 
autores son contradictorios, por falta de una mayor investigación en relación al principio activo, modo de acción y dosis (Franciosini et al., 2016).

\section{Conclusiones}

Bajo las condiciones de clima tropical de selva central, los tratamientos con antibióticos y probióticos presentaron similares pesos vivos, consumo de alimento y conversión alimenticia.

Las dietas alimenticias con orégano $1 \%$ generaron un menor peso vivo, pero similar consumo de alimento y conversión alimenticia en comparación a las dietas con antibióticos y probióticos.

\section{Referencias}

Ascensión, J. (2011). Efecto de la adición de una combinación de medicina natural (Orégano, cebolla, ajo, cilantro, epazote, manzanilla) vs promotores de crecimiento sobre los parámetros productivos de pollos de engorda. (tesis de pregrado). Universidad Veracruzana, Veracruz, México.

Chávez, L. A., López, A, \& Parra, J. E. (2016). El uso de Enterococcus faecium mejora los parámetros productivos en pollos de engorde. Revista de la Facultad de Medicina Veterinaria y de Zootecnia, 63(2), 113-123. doi.org/10.15446/rfmvz.v63n2.59358

Diario Oficial de la Unión Europea. (2003). Reglamento (CE) $\mathrm{N}^{\circ} \quad 1831 / 2003$ sobre los aditivos en la alimentación animal. Disponible en: https://www.boe.es/ doue/2003/268/L00029-00043.pdf

Franciosini, M.P., Casagrande-Proietti, P., Forte, C., Beghelli. D., Acuti, G. \& Zanichelli, D. (2016). Efectos de los extractos acuosos de orégano (Origanum vulgare L.) y romero (Rosmarinus officinalis L.) sobre el rendimiento del pollo de engorde, la función inmune y población microbiana intestinal. Investigación animal aplicada 44(1),474479. doi.org/10.1080/09712119.2015.1091 322

Gaggia, F., Mattarelli, P., \& Biavati, B. (2010). Probiotics and prebiotics in animal feeding for safe food production. International
Journal of Food Microbiology, 141, 15-28. doi.org/10.1016/j. ijfoodmicro.2010.02.031

Hernández, F., Madrid, J., Garcia, V., Orengo, J. \& Megías, M. D. (2004). Influence of two plant extracts on broilers performance, digestibility, and digestive organ size. Poultry Science, 83 (2), 169-174. doi. org/10.1093/ps/83.2.169

Jernigan, M. A., Miles, R. D. \& Arafa, A. S. (1985). Probiotics in poultry nutrition. A review. World Poultry Science Journal, 41, 99-105. doi.org/10.1079/WPS19850008

Lara y Lara, P.E., Itza, M.F., Aguilar, E. \& Sanginés, J.R. (2010). Harinas de hojas de plantas aromáticas como fitoterapéuticos en pollos de engorda. Pesquisa Agropecuária Brasileira, 45(3), 294-298. doi.org/10.1590/ S0100-204X2010000300009

Loddi, M. M., Gonzales, E., Takita, T. S., Mendes, A. A., de Oliveira, R. (2000). Effect of the use of probiotic and antibiotic on the performance and carcase quality of broilers. Journal Animal Science 29, 1124-1131. doi. org/10.1590/S1516-35982000000400025

Ministerio de Agricultura y Riego (2018). Boletín Estadístico Mensual de la Producción y Comercialización de Productos Avícolas. Disponible en: https://www.minagri.gob. pe/portal/boletin-estadistico-mensual-dela-produccion-y-comercializacion-avicola/ sector-avicola-2018

Osorio, A.M., Icochea, M.E., Reyna, P., Guzmán, O.A., Cazorla, F. \& Carcelén, F.D. (2010). Comparación del rendimiento productivo de pollos de carne suplementados con un probiótico versus un antibiótico. Revista de Investigaciones Veterinarias del Perú, 21(2), 219-222. doi.org/10.15381/rivep. v21i2.140

Quigley, E.M. (2010). Prebiotics and Probiotics. Modifying and mining the microbiota. Pharmacological Research, 61(3), 213-218. doi.org/10.1016/j.phrs.2010.01.004

Rincón, H.S., Pérez, C., Pérez, M.L., Briñez, W.J., Arzallus, A.M. Urdaneta, S.E. (2000). Efectos de la aplicación de bacterias lácticas y ácido láctico en la ganancia de peso y mortalidad en pollos de engorde. Revista Cientifica Luz, 10(4), 310-314. 
Salas, C. (2005). Efecto de la suplementación de paredes celulares de levadura sobre los rendimientos productivos de pollos de engorde. (tesis de pregrado). Universidad de Costa Rica, San José, Costa Rica
Watkins, B.A \& Kratzer, F.H. (1984). Drinking water treatment with commercial preparation of a concentrated Lactobacillus culture for broiler chickens. Poultry Science, 63(8), 1671-1673. doi.org/10.3382/ps.0631671 